\title{
Exchange coupling of a perpendicular ferromagnet to a half-metallic compensated ferrimagnet via a thin hafnium interlayer
}

Kiril Borisov, Gwenaël Atcheson, Gavin D'Arcy, Yong-Chang Lau, J. M. D. Coey, and Karsten Rode

Citation: Appl. Phys. Lett. 111, 102403 (2017);

View online: https://doi.org/10.1063/1.5001172

View Table of Contents: http://aip.scitation.org/toc/apl/111/10

Published by the American Institute of Physics

\section{Articles you may be interested in}

Spin-orbit torque induced magnetization switching in Co/Pt multilayers

Applied Physics Letters 111, 102402 (2017); 10.1063/1.5001171

Annealing effect on laser pulse-induced $\mathrm{THz}$ wave emission in $\mathrm{Ta} / \mathrm{CoFeB} / \mathrm{MgO}$ films

Applied Physics Letters 111, 102401 (2017); 10.1063/1.5001696

Enhancement of the spin-orbit torque in a Pt/Co system with a naturally oxidized Co layer Applied Physics Letters 111, 132404 (2017); 10.1063/1.4995292

Direct optical observation of spin accumulation at nonmagnetic metal/oxide interface Applied Physics Letters 111, 092402 (2017); 10.1063/1.4990113

Excitation of coherent propagating spin waves in ultrathin CoFeB film by voltage-controlled magnetic anisotropy Applied Physics Letters 111, 052404 (2017); 10.1063/1.4990724

Field-free spin-orbit torque switching of composite perpendicular CoFeB/Gd/CoFeB layers utilized for threeterminal magnetic tunnel junctions

Applied Physics Letters 111, 012402 (2017); 10.1063/1.4990994

\section{Scilight}

Sharp, quick summaries illuminating the latest physics research

\section{Sign up for FREE!}

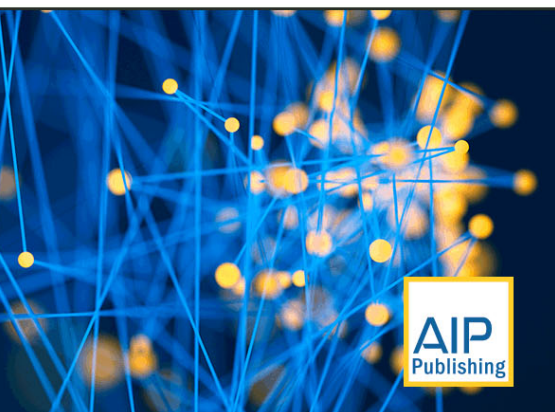




\title{
Exchange coupling of a perpendicular ferromagnet to a half-metallic compensated ferrimagnet via a thin hafnium interlayer
}

\author{
Kiril Borisov, ${ }^{1,2}$ Gwenaël Atcheson, ${ }^{1}$ Gavin D'Arcy, ${ }^{1}$ Yong-Chang Lau, ${ }^{1, a)}$ J. M. D. Coey, ${ }^{1}$ \\ and Karsten Rode ${ }^{1}$ \\ ${ }^{1}$ CRANN, AMBER and School of Physics, Trinity College, Dublin 2, Ireland \\ ${ }^{2}$ Institute of Nanotechnology, Karlsruhe Institute of Technology (KIT), D-76344 Eggenstein-Leopoldshafen, \\ Germany
}

(Received 8 May 2017; accepted 22 August 2017; published online 7 September 2017)

\begin{abstract}
A thin Hafnium film is shown to act both as an effective diffusion barrier for manganese at a thickness of $0.7 \mathrm{~nm}$ and as an effective exchange coupling layer in a sandwich structure with perpendicular magnetic anisotropy. The two magnetic layers are $\mathrm{Co}_{20} \mathrm{Fe}_{60} \mathrm{~B}_{20}$ and the low moment ferrimagnet $\mathrm{Mn}_{2} \mathrm{Ru}_{x} \mathrm{Ga}(\mathrm{MRG})$. The relative orientation of the magnetic layers changes sign at the compensation temperature of MRG. The exchange energy reaches $0.11 \mathrm{~mJ} \mathrm{~m}^{-2}$ for the thinnest Hf interlayers. Ruthenium, the usual metal of choice for coupling ferromagnetic layers in thin film heterostructures, cannot be used with the zero-moment half metal MRG because of Ru interdiffusion. Due to its large coercivity near compensation, the MRG can potentially act as an effective source of exchange pinning. Published by AIP Publishing. [http://dx.doi.org/10.1063/1.5001172]
\end{abstract}

Spin electronics has influenced the information revolution through technologies such as affordable high-density magnetic storage and high-speed nonvolatile magnetic memory, taking advantage of the giant magnetoresistance or tunneling magnetoresistance (GMR or TMR) effects to read and store information. In the magnetic thin film stacks employed as field sensors or magnetic switches, it is useful to be able to control the magnetic coupling between adjacent layers. Direct contact between an antiferromagnetic layer and a ferromagnetic layer can lead to exchange bias of the latter, enabling it to serve as a pinned or reference layer in any spin valve sandwich structure. Stacks may also make use of the interlayer exchange coupling $^{1,2}$ to form synthetic antiferromagnets (SAFs) where two ferromagnetic layers are coupled antiferromagnetically via a thin layer of nonmagnetic metal in a structure that creates no stray field. The exchange coupling can be further engineered to form structures that enable demonstration of ultrafast Néel domain wall motion ${ }^{3}$ and deterministic spin orbit torqueinduced switching of a perpendicular ferromagnet without an external field. ${ }^{4,5}$ The sign of the exchange usually oscillates with the nonmagnetic spacer thickness, and Ru has been found to be the most effective. ${ }^{1}$ A peak in the antiferromagnetic coupling is found for a ruthenium thickness of $0.9 \mathrm{~nm}$, where the exchange energy reaches $0.11 \mathrm{~mJ} \mathrm{~m}^{-2}$. An early systematic study by Parkin found no coupling between $3 d$ ferromagnetic layers for the $d^{2}$ transition metals $\mathrm{Ti}, \mathrm{Zr}$, or $\mathrm{Hf}^{2}$

An alternative to using an exchange-biased SAF to pin the reference layer is to exchange-couple it to a low-moment uniaxial ferrimagnet that is close to compensation. ${ }^{6}$ This could produce the necessary exchange bias, whilst adding magnetic mass to stabilise ultra-thin $\mathrm{CoFeB}$ layers in perpendicular magnetic tunnel junctions (MTJs). ${ }^{7}$ The uniaxial anisotropy $K_{1}$ ensures that, as the magnetisation $M_{s}$ of the ferrimagnet approaches zero at the compensation temperature $\left(T_{\text {comp }}\right)$, the anisotropy field $2 K_{1} / \mu_{0} M_{s}$ diverges and the coercivity increases

\footnotetext{
a) Present address: Department of Physics, The University of Tokyo, Bunkyo, Tokyo 113-0033, Japan.
}

significantly. ${ }^{8,9}$ Thin films of the recently discovered compensated ferrimagnetic half-metal $\mathrm{Mn}_{2} \mathrm{Ru}_{x} \mathrm{Ga}(\mathrm{MRG})^{10}$ have been shown to exhibit high spin-polarisation and a large coercivity near compensation, ${ }^{8,11}$ due to their perpendicular magnetic anisotropy (PMA) caused by biaxial substrate-induced strain of the cubic Heusler structure. Similar materials have shown magnetic resonance in the low THz range. ${ }^{12,13}$ MRG has a Heuslertype structure as shown in Fig. 1(a), with Mn on $4 a$ and $4 c$ sites, $\mathrm{Ga}$ on $4 b$ sites, and $\mathrm{Ru}$ on most of the $4 d$ sites. ${ }^{10,14}$ Some MnGa antisite substitution is present in the structure, ${ }^{14}$ which leads to a spin gap at the Fermi level. ${ }^{15}$ Here, we show that the MRG $4 c$ sublattice can be coupled antiferromagnetically to an adjacent perpendicular $\mathrm{CoFeB}$ layer via a thin $\mathrm{Hf}$ interlayer, and the effect can be used to produce exchange pinning.

The $\mathrm{Mn}_{2} \mathrm{Ru}_{0.7} \mathrm{Ga}$ used in this study has $T_{\text {comp }} \approx 240 \mathrm{~K}$, so data can be conveniently collected at temperatures above
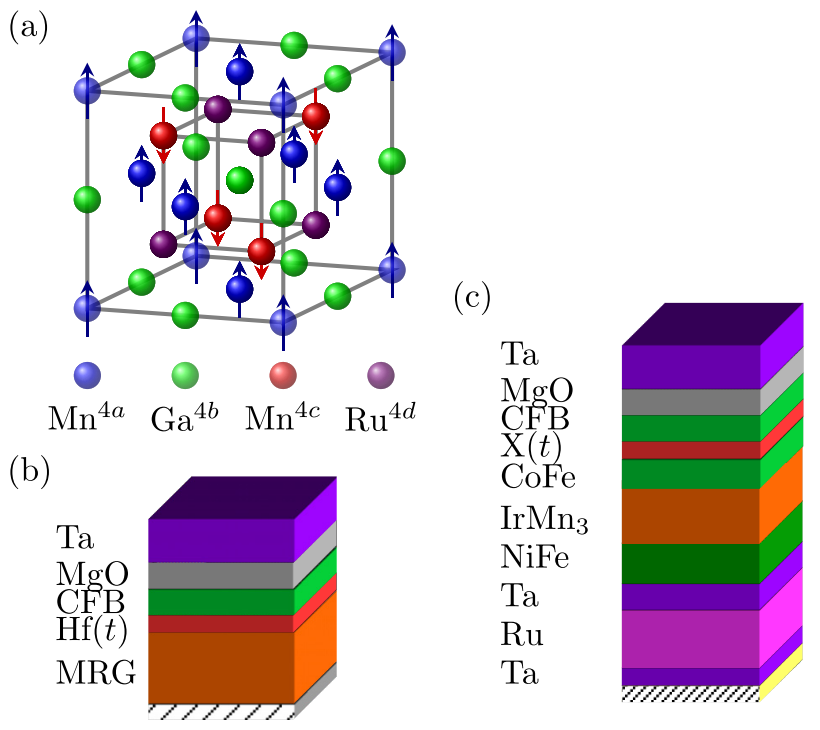

FIG. 1. (a) Crystal structure of $\mathrm{Mn}_{2} \mathrm{Ru}_{x} \mathrm{Ga}$. (b) Fully perpendicular thin film stack used to investigate exchange coupling via Hf and (c) an in-plane magnetised reference stack without $\mathrm{MRG}, \mathrm{X}=\mathrm{Hf}$ or $\mathrm{Ru}$. The Hf thickness, $t$, was varied from 0.4 to $1.6 \mathrm{~nm}$. 
and below compensation. Stacks of $\mathrm{MgO} / / \mathrm{MRG}(35) / \mathrm{Hf}(t) /$ $\mathrm{CFB}(1) / \mathrm{MgO}(1.4) / \mathrm{Ta}(1)$ (thicknesses in $\mathrm{nm}$ ), where $t=0.3$, $0.4,0.5,0.6,0.7,0.8,0.9,1.1,1.2$, and $1.6 \mathrm{~nm}$, were grown on $10 \times 10 \mathrm{~mm}^{2}$ single-crystal $\mathrm{MgO}(100)$ substrates using a Shamrock magnetron sputter deposition tool. Here, CFB denotes $\mathrm{Co}_{20} \mathrm{Fe}_{60} \mathrm{~B}_{20}$ (nominal target composition). The MRG was DC co-sputtered from stoichiometric $\mathrm{Mn}_{2} \mathrm{Ga}$ and $\mathrm{Ru}$ targets, at a substrate temperature of $380^{\circ} \mathrm{C} .{ }^{8,10}$ After cooling, $\mathrm{Hf}, \mathrm{CFB}, \mathrm{MgO}$, and Ta were then sputtered at room temperature. Stacks were post-annealed under a vacuum of $\sim 10^{-6}$ mbar in an applied perpendicular magnetic field of $800 \mathrm{mT}$ at $300^{\circ} \mathrm{C}$ for $30 \mathrm{~min}$ to enhance the PMA of CFB. In-plane-magnetised reference stacks $^{16}$ of composition $\mathrm{Ta}(5) / \mathrm{Ru}(10) / \mathrm{Ta}(5) / \mathrm{Ni}_{81} \mathrm{Fe}_{19}(5) / \mathrm{Ir}_{22} \mathrm{Mn}_{78}(10) / \mathrm{Co}_{90} \mathrm{Fe}_{10}(2.4) /$ $\mathrm{X}(t) / \mathrm{CFB}(3) / \mathrm{MgO}(1.4) / \mathrm{Ta}(5)$, where $\mathrm{X}=\mathrm{Hf}$ or $\mathrm{Ru}$ and $t=0.5,0.7,0.9$, and $1.0 \mathrm{~nm}$, were deposited on thermally oxidised $20 \times 20 \mathrm{~mm}^{2} \mathrm{Si} / \mathrm{SiO}_{2}$ coupons at room temperature. The reference stacks were post-annealed in an in-plane applied field at $350^{\circ} \mathrm{C}$ for $60 \mathrm{~min}$ to set the exchange bias at the $\mathrm{IrMn} / \mathrm{CoFe}$ interface. The stacks are illustrated in Figs. 1 (b) and $1(\mathrm{c})$.

The anomalous Hall effect (AHE) was measured using the 4-point van der Pauw method, with indium contacts, an applied current of $1 \mathrm{~mA}$, and an applied magnetic field of up to $4 \mathrm{~T}$. Additional Hall measurements up to $14 \mathrm{~T}$ were carried out in a Quantum Design PPMS system. Room temperature magnetisation measurements with magnetic field applied inplane or perpendicular to the films were carried out using a Quantum Design SQUID magnetometer with a maximum field of $5 \mathrm{~T}$.

If we are to use MRG, more generally, the Mncontaining Heusler alloys, in an exchange coupled system, we have to consider three factors when deciding what metal interlayer to use: Does it prevent interdiffusion between the MRG and the adjacent layers? ${ }^{17}$ Does it maintain perpendicular anisotropy of the overlayer? ${ }^{18-20}$ Does it provide sufficient coupling between the MRG and an adjacent ferromagnetic layer? ${ }^{21}$ An ideal material will do all three, but the selection is limited. Initially, a number of candidates were chosen, namely, $\mathrm{W}, \mathrm{Zr}, \mathrm{Ru}, \mathrm{Mo}, \mathrm{Hf}, \mathrm{Ta}$, and TiN. Some of them were effective at blocking diffusion between layers ${ }^{22}$ which is necessary to preserve spin-polarisation and device integrity; ${ }^{17}$ however, only Mo, Hf, and Ta also maintained the PMA of the CFB overlayer, and of these, Hf was the only one to demonstrate strong effective exchange coupling between MRG and CFB as well. An order of magnitude smaller effect was found with the thinnest Ta $(0.4 \mathrm{~nm})$. Ruthenium, the usual material of choice for exchange coupling ferromagnetic films, is unsuitable due to the lack of PMA in $\mathrm{Ru} / \mathrm{CFB} / \mathrm{MgO}$ heterostructures ${ }^{23}$ and the incorporation of $\mathrm{Ru}$ into the MRG layer, which alters its magnetic properties.

The $\mathrm{Mn}_{2} \mathrm{Ru}_{0.7} \mathrm{Ga}$ composition with $T_{\text {comp }} \approx 240 \mathrm{~K}$ has a room-temperature magnetisation of $40 \mathrm{kA} \mathrm{m}^{-1}$ and coercivity $\mu_{0} H_{c}=0.7 \mathrm{~T}$. Small variations in composition and strain between deposition runs result in minor changes in $4 a$ and $4 c$ magnetisations between sample sets, which may change the coercivity by more than $1 \mathrm{~T}$ in the vicinity of $T_{\text {comp }} \cdot{ }^{14,24}$ For $t \leq 0.3 \mathrm{~nm}$, there was no PMA for the CFB and no exchange coupling was observed for any of the seven interlayer

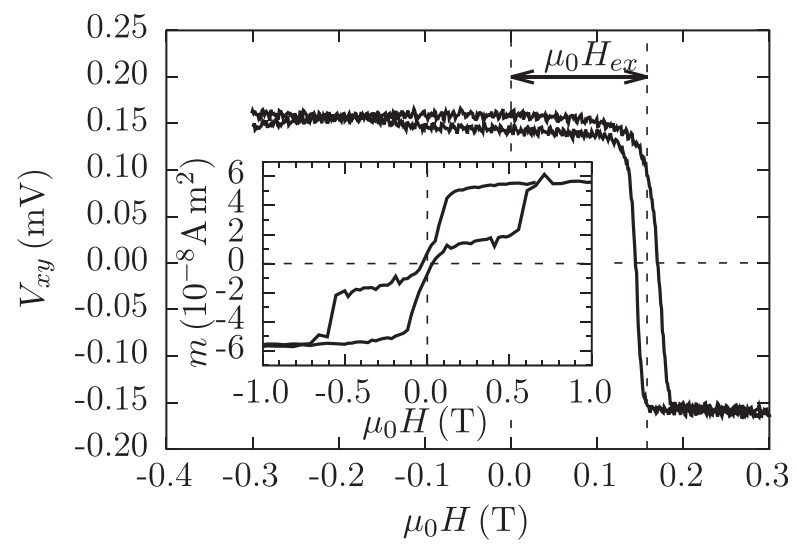

FIG. 2. Minor AHE loop of $\mathrm{CoFeB}$ for the stack of Fig. 1(b) with a $0.4 \mathrm{~nm}$ Hf interlayer measured above compensation, showing strong antiferromagnetic exchange coupling of the $\mathrm{Mn}^{4 c}$ and CFB layers after saturating in the negative field. The inset shows major SQUID magnetisation loops for a stack of Fig. 1(b) with $t=0.4 \mathrm{~nm}$. Note that the SQUID loop shown was measured below $T_{\text {comp }}$ of MRG. At remanence, the net moments are parallel above $T_{\text {comp }}$ and anti-parallel below $T_{\text {comp }}$.

materials. Due to the demagnetising field of the $\mathrm{CoFeB}$ with easy plane anisotropy, we are unable to reliably evaluate the exchange coupling for a $\mathrm{Hf}$ thickness of $0.3 \mathrm{~nm}$ or lower.

The effect of exchange coupling is nicely illustrated for a Hf interlayer with $t=0.4 \mathrm{~nm}$ in Fig. 2, where the CFB layer is perpendicular and minor hysteresis loops can be readily measured using the AHE. The narrow hysteresis loop of the CFB with a coercivity of $16 \mathrm{mT}$ is strongly exchange shifted by $\mu_{0} H_{e x}=175 \mathrm{mT}$ in the direction opposite to the saturation field. These measurements have been repeated for greater Hf thickness, and $\mu_{0} H_{e x}$ decreases monotonically with $t$, falling to zero at $t \approx 1.0 \mathrm{~nm}$ (Fig. 3). Note that the exchange shift of the loop in Fig. 2, observed after saturation in $-4 \mathrm{~T}$, is towards positive fields. This shows that the weaker (above $T_{\text {comp }}$ ) $4 c$ sublattice is coupled antiferromagnetically via the Hf layer to the $\mathrm{CFB}$, as confirmed by the data in Fig. 4(a). Note that the $4 c$ sublattice is aligned antiparallel to the net MRG magnetisation when $T>T_{\text {comp }}$.

The complete hysteresis loop for $t=0.4 \mathrm{~nm}$ is shown in the inset of Fig. 2, and the congruent values of $\mu_{0} H_{e x}$ measured by AHE and SQUID magnetometry are compared in Fig. 3. The greatest exchange shift $\mu_{0} H_{e x}=174 \mathrm{mT}$ is found for the interlayer with $t=0.4 \mathrm{~nm}$, and the shift has disappeared at $t=0.9 \mathrm{~nm}$. The exchange energy $J_{e x}$ is estimated

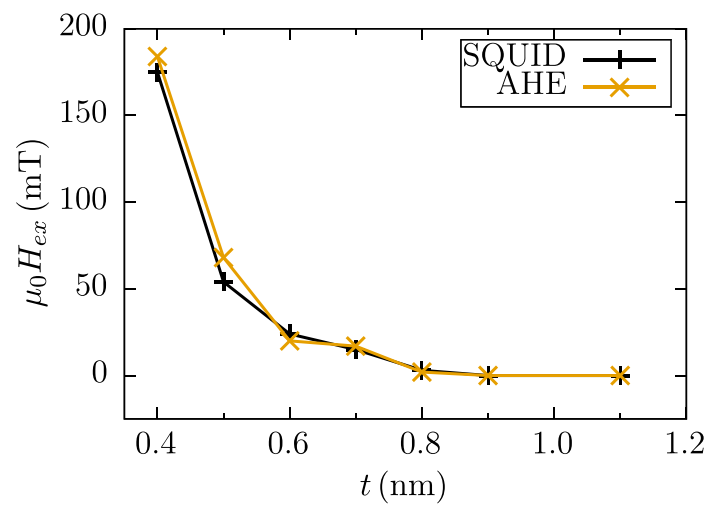

FIG. 3. Exchange shifts measured by SQUID or AHE as a function of the thickness of the Hf spacer layer. 


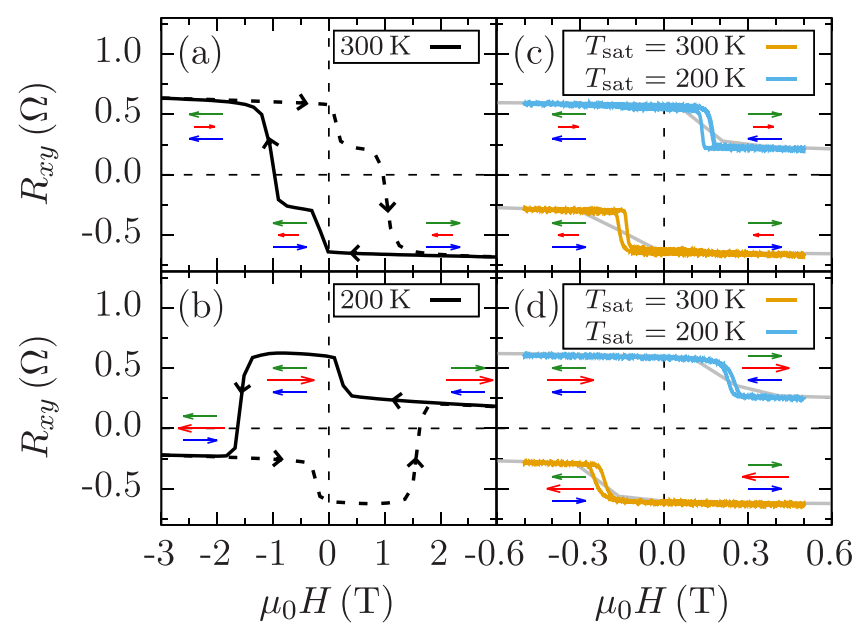

FIG. 4. Major (left) and minor (right) AHE loops for the stack of Fig. 1(b) with $t=0.4 \mathrm{~nm}$. The direction of field sweep is indicated by black arrows. Data shown in the top and bottom row are at $T=300$ and $200 \mathrm{~K}$ ( $>$ and $<T_{\text {comp }}$ ), respectively. The small arrows indicate the relative directions of the CFB (in green), MRG $4 c$ sublattice (red), and MRG $4 a$ sublattice (blue). The arrows in the plateau regions show the configuration after the CFB has switched. The difference between blue and red arrows indicates the net moment of the MRG. The faint grey line in (c) and (d) denotes the trace of the major loop. The data establish that the relative alignment of MRG and CFB changes when MRG is saturated below or above $T_{\text {comp }}$.

as $\mu_{0} H_{e x} M_{\mathrm{CFB}} t_{\mathrm{CFB}}{ }^{25,26}$ Taking $M_{\mathrm{CFB}}=0.6 \mathrm{MA} \mathrm{m}^{-1}$, the maximum value of $J_{e x}$ is $0.11 \mathrm{~mJ} \mathrm{~m}^{-2}$. This is comparable to the antiferromagnetic exchange coupling for $\mathrm{CoFe} / \mathrm{CFB}$ layers separated by a $\mathrm{Ru}$ interlayer of thickness $t_{\mathrm{Ru}} \approx$ $0.9 \mathrm{~nm},{ }^{27}$ which corresponds to the easily attainable second AFM peak of $\mathrm{Ru}$. Reports on the first AFM peak showing stronger coupling $\left(2.2-5 \mathrm{~mJ} \mathrm{~m}^{-2}\right)$ with $t_{\mathrm{Ru}}=0.4$ to $0.5 \mathrm{~nm}$ are scarce. ${ }^{1,28,29}$

In Figs. 4(a) and 4(b), full AHE loops are shown above ( $300 \mathrm{~K})$ and below $(200 \mathrm{~K}) T_{\text {comp. The applied field sequence }}$ after saturation was +-+ . We analyse the behaviour in terms of the relative orientation of the CFB and the MRG $4 c$ sublattice, which is the one that contributes to the MRG density of states at the Fermi level. ${ }^{14}$ At $300 \mathrm{~K}\left(T>T_{\text {comp }}\right), 4 c$ is the weaker sublattice and hence it is antiparallel to the net MRG magnetisation. CFB switches after passing zero applied field [in the third quadrant Fig. 4(a)], and the alignment of the CFB and the net MRG moment changes. In a larger field of $-1.0 \mathrm{~T}$, the MRG flips. At $200 \mathrm{~K}$ [Fig. 4(b)], the $4 c$ sublattice is the stronger one and is parallel to the net MRG magnetisation. CFB now switches in the first quadrant before reaching zero applied field, corresponding to antiparallel alignment with the net MRG moment and the $4 c$ sublattice. The MRG contribution to the AHE magnitude changes sign when we cross $T_{\text {comp }}$, as we have shown previously. ${ }^{8}$

In order to highlight the relative antiparallel alignment of the $4 c$ sublattice moment and the CFB, we measured minor loops of CFB in the range $\pm 0.5 \mathrm{~T}$, both above and below $T_{\text {comp }}$ [Figs. 4(c) and 4(d)] after first saturating in a positive field. Two measurements were made in each case: First, after saturation at the measurement temperature and second, after saturation on the other side of $T_{\text {comp }}$ and heating [Fig. 4(c)] or cooling [Fig. 4(d)] back to the measurement temperature in zero field. When crossing $T_{\text {comp }}$ in zero field, the net MRG magnetisation, $M$, changes from positive to negative while the direction of the $4 c$ sublattice, $M_{4 c}$, is preserved, and therefore, all four possible combinations of the orientations of $M$ and $M_{4 c}$ are accessible. We find that the $\mathrm{Mn}^{4 c}-\mathrm{CFB}$ coupling is always antiferromagnetic, but the alignment of CFB is parallel with respect to the net moment above $T_{\text {comp }}$ and antiparallel below.

Finally, we show in Fig. 5 the SQUID loops for the inplane reference stack of Fig. 1(b) where a CoFe layer is separated by a hafnium or ruthenium interlayer from the upper CFB layer. The data show clear evidence of antiferromagnetic exchange coupling for $\mathrm{Ru}(0.9 \mathrm{~nm})$ and strong ferromagnetic coupling when the spacer is $\operatorname{Hf}(0.7 \mathrm{~nm})$, in contrast with early reports. ${ }^{2}$

Unlike ruthenium, ${ }^{1}$ where the sign of the exchange coupling oscillates with the interlayer thickness with a period of about $1.1 \mathrm{~nm}$, reminiscent of the Ruderman-Kittel-Kasuya-Yosida (RKKY) interaction, the exchange coupling through hafnium is always antiferromagnetic in the case of $\mathrm{MRG}(4 c) / \mathrm{Hf} / \mathrm{CFB}$ and ferromagnetic for the $\mathrm{CoFe} / \mathrm{Hf} / \mathrm{CFB}$ stack, and it decreases monotonically to zero with increasing interlayer thickness. The coupling is the strongest for the very thin $0.4 \mathrm{~nm}$ films that are only two atomic layers thick. A possible mechanism for the MRG/CFB coupling via $\mathrm{Hf}$ is hybridization of the unpolarized Hf $6 s^{2} 5 d^{2}$ conduction electrons with the spin polarized $4 c$ sub-band at the Fermi surface of MRG. This polarizes the first few layers of Hf, rather as the $5 d 6 s$ bands of the rare-earth metals are polarized by the $4 f$ core. Coupling between an atom with a nearly full $d$ band (Co) and the electrons of a nearly empty $d$ band (Hf) is antiferromagnetic. ${ }^{30,31}$ The CFB/MRG alignment becomes parallel when $4 c$ is the weaker sublattice, above $T_{\text {comp }}$, and antiparallel when $4 c$ becomes the stronger sublattice, below $T_{\text {comp. }}$. We observed from Fig. 4 that the alignment changes sign at $T_{\text {comp }}$ in just the sense that is predicted. For the reference $\mathrm{CoFe} / \mathrm{Hf} / \mathrm{CFB}$ stack, the two antiferromagnetic interactions via $\mathrm{Hf}$ lead to an overall parallel alignment as observed. A similar effect has been observed in $L 1_{0}-\mathrm{MnGa} / \mathrm{Fe}_{1-x} \mathrm{Co}_{x}{ }^{32}$ as a function of $x$ which was attributed to the reversal of the interfacial spin polarisation.

Another mechanism that could produce the same effect, with a sign change at compensation, is exchange coupling via pinholes in the thin interlayer. The $0.4 \mathrm{~nm} \mathrm{Hf}$ layer is unlikely to be entirely defect-free, but this explanation is

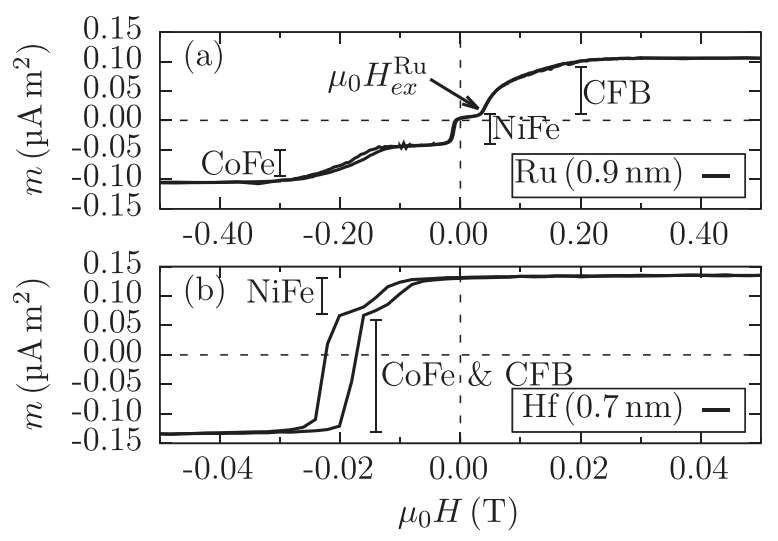

FIG. 5. SQUID magnetometry of the in-plane reference stack of Fig. 1(c). (a) Antiferromagnetic coupling with $t_{\mathrm{Ru}}=0.9 \mathrm{~nm}$. The exchange shift is $\mu_{0} H_{e x}^{\mathrm{Ru}} \approx 33 \mathrm{mT}$ which is indicated with an arrow. (b) Strong ferromagnetic coupling with $t_{\mathrm{Hf}}=0.7 \mathrm{~nm}$. The exchange bias is $\mu_{0} H_{e x}^{\mathrm{IrMn}} \approx 22 \mathrm{mT}$. 
implausible for several reasons. First, it should apply to any interlayer with pinholes. Hf is the only one of the seven metals we have studied which exhibits strong exchange coupling. Furthermore, no exchange was observed for $t_{\mathrm{Hf}}=$ $0.3 \mathrm{~nm}$ where the case for pinholes would be even stronger. Finally, when CFB is deposited directly on MRG, no exchange is observed in the crossed anisotropy system. We can therefore discount this explanation.

Exchange coupling has been demonstrated before between ferrimagnet and ferrimagnet ${ }^{21,33,34}$ and ferrimagnet and ferromagnet ${ }^{35-40}$ and in frustrated systems. ${ }^{41}$ The advantages of our approach are the MRG high ordering temperature $\left(T_{\mathrm{C}} \approx 550 \mathrm{~K}\right)^{10}$ and the broad tunability of the compensation temperature $(2$ to $400 \mathrm{~K}){ }^{8}{ }^{8}$ The use of a single layer of MRG or some other near-zero-moment ferrimagnet close to compensation can in principle replace the standard SAF reference layer in an all-perpendicular MTJ, such as buffer-layer/[Co/Pt $]_{n} / \mathrm{Ru} /[\mathrm{Co} / \mathrm{Pt}]_{n} / \mathrm{Ta}$ or $\mathrm{W} / \mathrm{CFB},{ }^{29,42}$ provided that the uniaxial anisotropy is positive so that the anisotropy field diverges when $M_{s} \rightarrow 0$ as discussed above. The difficulty with using MRG or any Mn-based ferrimagnet in direct contact with a ferromagnet or a tunnel barrier such as $\mathrm{MgO}$ is manganese diffusion into the overlayer. ${ }^{11,17,43}$ Here, we have demonstrated the feasibility of a simple bcctextured MRG/Hf/CFB reference structure that can be grown directly on $\mathrm{MgO}$ as an alternative. A fully bcc-textured MTJ stack with a robust reference layer can therefore be realised. Furthermore, the use of MRG with high spin polarisation in conjunction with a Hf interlayer with relatively high spin transparency ${ }^{19}$ may also be advantageous for enhancing the spin-torque efficiency of the MTJ.

We previously demonstrated that our compensated halfmetallic Heusler alloy MRG combines the advantageous properties of both ferro- and antiferro-magnets without their limitations. ${ }^{8,10-12,14}$ It can be used as a source of spinpolarised currents, is immune to external fields, and produces no stray field of its own. In this work, we have demonstrated another application: as a reference layer in a spin electronic stack, either alone or as a pinning material, thereby greatly reducing the complexity of the stack.

K.B. acknowledges financial support from Science Foundation Ireland within SSPP (11/SIRG/I2130). This work was partly supported by Science Foundation Ireland under Grant Nos. 13/ERC/12561 and 12/RC/2278 (AMBER). This project has received funding from the European Union's Horizon 2020 research and innovation programme under Grant Agreement No. DLV-737038 (TRANSPIRE).

${ }^{1}$ S. S. P. Parkin, N. More, and K. P. Roche, Phys. Rev. Lett. 64, 2304 (1990).

${ }^{2}$ S. S. P. Parkin, Phys. Rev. Lett. 67, 3598 (1991).

${ }^{3}$ S.-H. Yang, K.-S. Ryu, and S. Parkin, Nat. Nanotechnol. 10, 221 (2015).

${ }^{4}$ Y.-C. Lau, D. Betto, K. Rode, J. M. D. Coey, and P. Stamenov, Nat. Nanotechnol. 11, 758 (2016).

${ }^{5}$ S. Fukami, C. Zhang, S. DuttaGupta, A. Kurenkov, and H. Ohno, Nat. Mater. 15, 535 (2016).

${ }^{6}$ A. K. Nayak, M. Nicklas, S. Chadov, P. Khuntia, C. Shekhar, A. Kalache, M. Baenitz, Y. Skourski, V. K. Guduru, A. Puri, U. Zeitler, J. M. D. Coey, and C. Felser, Nat. Mater. 14, 679 (2015).
${ }^{7}$ S. Ikeda, K. Miura, H. Yamamoto, K. Mizunuma, H. D. Gan, M. Endo, S. Kanai, J. Hayakawa, F. Matsukura, and H. Ohno, Nat. Mater. 9, 721 (2010).

${ }^{8}$ N. Thiyagarajah, Y.-C. Lau, D. Betto, K. Borisov, J. M. D. Coey, P. Stamenov, and K. Rode, Appl. Phys. Lett. 106, 122402 (2015).

${ }^{9}$ D. Betto, Y.-C. Lau, K. Borisov, K. Kummer, N. B. Brookes, P. Stamenov, J. M. D. Coey, and K. Rode, Phys. Rev. B 96, 024408 (2017).

${ }^{10}$ H. Kurt, K. Rode, P. Stamenov, M. Venkatesan, Y.-C. Lau, E. Fonda, and J. M. D. Coey, Phys. Rev. Lett. 112, 027201 (2014).

${ }^{11}$ K. Borisov, D. Betto, Y.-C. Lau, C. Fowley, A. Titova, N. Thiyagarajah, G. Atcheson, J. Lindner, A. M. Deac, J. M. D. Coey, P. Stamenov, and K. Rode, Appl. Phys. Lett. 108, 192407 (2016).

${ }^{12}$ N. Awari, S. Kovalev, C. Fowley, K. Rode, R. A. Gallardo, Y.-C. Lau, D. Betto, N. Thiyagarajah, B. Green, O. Yildirim, J. Lindner, J. Fassbender, J. M. D. Coey, A. M. Deac, and M. Gensch, Appl. Phys. Lett. 109, 032403 (2016).

${ }^{13}$ S. Mizukami, A. Sugihara, S. Iihama, Y. Sasaki, K. Z. Suzuki, and T. Miyazaki, Appl. Phys. Lett. 108, 012404 (2016).

${ }^{14}$ D. Betto, N. Thiyagarajah, Y.-C. Lau, C. Piamonteze, M.-A. Arrio, P. Stamenov, J. M. D. Coey, and K. Rode, Phys. Rev. B 91, 094410 (2015).

${ }^{15}$ M. Žic, K. Rode, N. Thiyagarajah, Y.-C. Lau, D. Betto, J. M. D. Coey, S. Sanvito, K. J. O’Shea, C. A. Ferguson, D. A. MacLaren, and T. Archer, Phys. Rev. B 93, 140202 (2016).

${ }^{16}$ S. Yuasa and D. D. Djayaprawira, J. Phys. D: Appl. Phys. 40, R337 (2007).

${ }^{17}$ J. Hayakawa, S. Ikeda, Y. M. Lee, F. Matsukura, and H. Ohno, Appl. Phys. Lett. 89, 232510 (2006).

${ }^{18}$ T. Liu, J. W. Cai, and L. Sun, AIP Adv. 2, 032151 (2012).

${ }^{19}$ C.-F. Pai, M.-H. Nguyen, C. Belvin, L. H. Vilela-Leão, D. C. Ralph, and R. A. Buhrman, Appl. Phys. Lett. 104, 082407 (2014).

${ }^{20}$ Y.-W. Oh, K.-D. Lee, J.-R. Jeong, and B.-G. Park, J. Appl. Phys. 115, 17C724 (2014).

${ }^{21}$ F. Radu, R. Abrudan, I. Radu, D. Schmitz, and H. Zabel, Nat. Commun. 3, 715 (2012).

${ }^{22}$ The diffusion profile has been recorded by Time-of-Flight Secondary Ion Mass Spectroscopy (unpublished).

${ }^{23}$ D. C. Worledge, G. Hu, D. W. Abraham, J. Z. Sun, P. L. Trouilloud, J. Nowak, S. Brown, M. C. Gaidis, E. J. O'Sullivan, and R. P. Robertazzi, Appl. Phys. Lett. 98, 022501 (2011).

${ }^{24}$ K. E. Siewierska, G. Atcheson, K. Borisov, M. Venkatesan, K. Rode, and J. M. D. Coey, IEEE Trans. Magn. PP, 1 (2017).

${ }^{25}$ W. H. Meiklejohn and C. P. Bean, Phys. Rev. 102, 1413 (1956).

${ }^{26}$ W. H. Meiklejohn, J. Appl. Phys. 33, 1328 (1962).

${ }^{27}$ J. H. Jung, S. H. Lim, and S. R. Lee, Appl. Phys. Lett. 101, 242403 (2012).

${ }^{28}$ Y. C. Lau, K. Oguz, K. Rode, and J. M. D. Coey, Eur. Phys. J. B 86, 110 (2013).

${ }^{29}$ K. Yakushiji, H. Kubota, A. Fukushima, and S. Yuasa, Appl. Phys. Express 8, 083003 (2015).

${ }^{30}$ I. A. Campbell, J. Phys. C: Solid State Phys. 2, 1338 (1969).

${ }^{31}$ I. A. Campbell, J. Phys. F: Met. Phys. 2, L47 (1972).

${ }^{32}$ Q. L. Ma, S. Mizukami, T. Kubota, X. M. Zhang, Y. Ando, and T. Miyazaki, Phys. Rev. Lett. 112, 157202 (2014).

${ }^{33}$ S. Mangin, F. Montaigne, and A. Schuhl, Phys. Rev. B 68, 140404 (2003).

${ }^{34}$ B. Hebler, P. Reinhardt, G. L. Katona, O. Hellwig, and M. Albrecht, Phys. Rev. B 95, 104410 (2017).

${ }^{35}$ C. Schubert, B. Hebler, H. Schletter, A. Liebig, M. Daniel, R. Abrudan, F. Radu, and M. Albrecht, Phys. Rev. B 87, 054415 (2013).

${ }^{36}$ B. Hebler, S. Böttger, D. Nissen, R. Abrudan, F. Radu, and M. Albrecht, Phys. Rev. B 93, 184423 (2016).

${ }^{37}$ S. M. Watson, T. Hauet, J. A. Borchers, S. Mangin, and E. E. Fullerton, Appl. Phys. Lett. 92, 202507 (2008).

${ }^{38}$ S. Mangin, T. Hauet, P. Fischer, D. H. Kim, J. B. Kortright, K. Chesnel, E. Arenholz, and E. E. Fullerton, Phys. Rev. B 78, 024424 (2008).

${ }^{39}$ M. Ungureanu, K. Dumesnil, C. Dufour, N. Gonzalez, F. Wilhelm, A. Smekhova, and A. Rogalev, Phys. Rev. B 82, 174421 (2010).

${ }^{40}$ S. Romer, M. A. Marioni, K. Thorwarth, N. R. Joshi, C. E. Corticelli, H. J. Hug, S. Oezer, M. Parlinska-Wojtan, and H. Rohrmann, Appl. Phys. Lett. 101, 222404 (2012).

${ }^{41}$ P. Henelius, T. Lin, M. Enjalran, Z. Hao, J. G. Rau, J. Altosaar, F. Flicker, T. Yavors'kii, and M. J. P. Gingras, Phys. Rev. B 93, 024402 (2016).

${ }^{42}$ L. Cuchet, B. Rodmacq, S. Auffret, R. C. Sousa, C. Ducruet, and B. Dieny, Appl. Phys. Lett. 103, 052402 (2013).

${ }^{43}$ T. Kubota, S. Mizukami, D. Watanabe, F. Wu, X. Zhang, H. Naganuma, M. Oogane, Y. Ando, and T. Miyazaki, J. Appl. Phys. 110, 013915 (2011). 